\title{
Depression, Anxiety, Cannabis and Opioid Use and Complementary Integrative Health Practices in Women with Chronic Pain
}

\author{
D. Svikis ${ }^{1,2}$, L. Phipps' ${ }^{2}$, J. Sadicario ${ }^{1}$, A. Parlier ${ }^{1}$, J. Jacob², N. Karjane ${ }^{3}$, A. Gataric ${ }^{3}$ \\ 'Virginia Commonwealth University, Department of Psychology, Richmond, Virginia USA. \\ ${ }^{2}$ Virginia Commonwealth University, Institute for Women's Health, Richmond, Virginia USA. \\ ${ }^{3}$ Virginia Commonwealth University, Department of Obstetrics and Gynecology, Richmond, Virginia USA.
}

\section{Objective:}

To examine relationships between chronic pain, depression, anxiety, opioid and cannabis use and complementary/integrative health practices in women.

\section{Background:}

- Women report more chronic pain (ChrP) conditions and are more likely to be prescribed opioids than males ${ }^{1}$.

- Concern about opioid misuse has prompted exploration of other treatment options. ${ }^{2}$

- ChrP is the leading cause for use of complementary health practices (CHP) and about one-third of adults in USA have used CHP for this purpose. ${ }^{1}$

- Research has found 30 to $50 \%$ of people with chronic pain also report depression or anxiety. ${ }^{3}$

- Cannabis has been used as substitute for opioids, especially among those reporting pain, anxiety, and/or depression ${ }^{4}$.

\section{Materials and Methods:}

- Participants were 210 women recruited from two OB/Gyn practices

- Completed anonymous computer-based health survey focused on demographics, pain (chronic and acute); CIHP and opioid use as well as standardized measures of depression (PHQ) and anxiety (GAD-7).

- Comparisons were made using t-tests for continuous and chisquare for categorical variables.

\section{Results:}

- Sample mean age $=38.5$ yrs. and $45 \%$ were Caucasian.

- Chronic pain (ChrP) was reported by $42 \%$ of the sample, and over half $(58.6 \%)$ of ChrP women were prescribed an opioid.

- Over one third (37\%) reported using CHP for pain management.

Fig 1. PHQ Depression and GAD Anxiety Scores

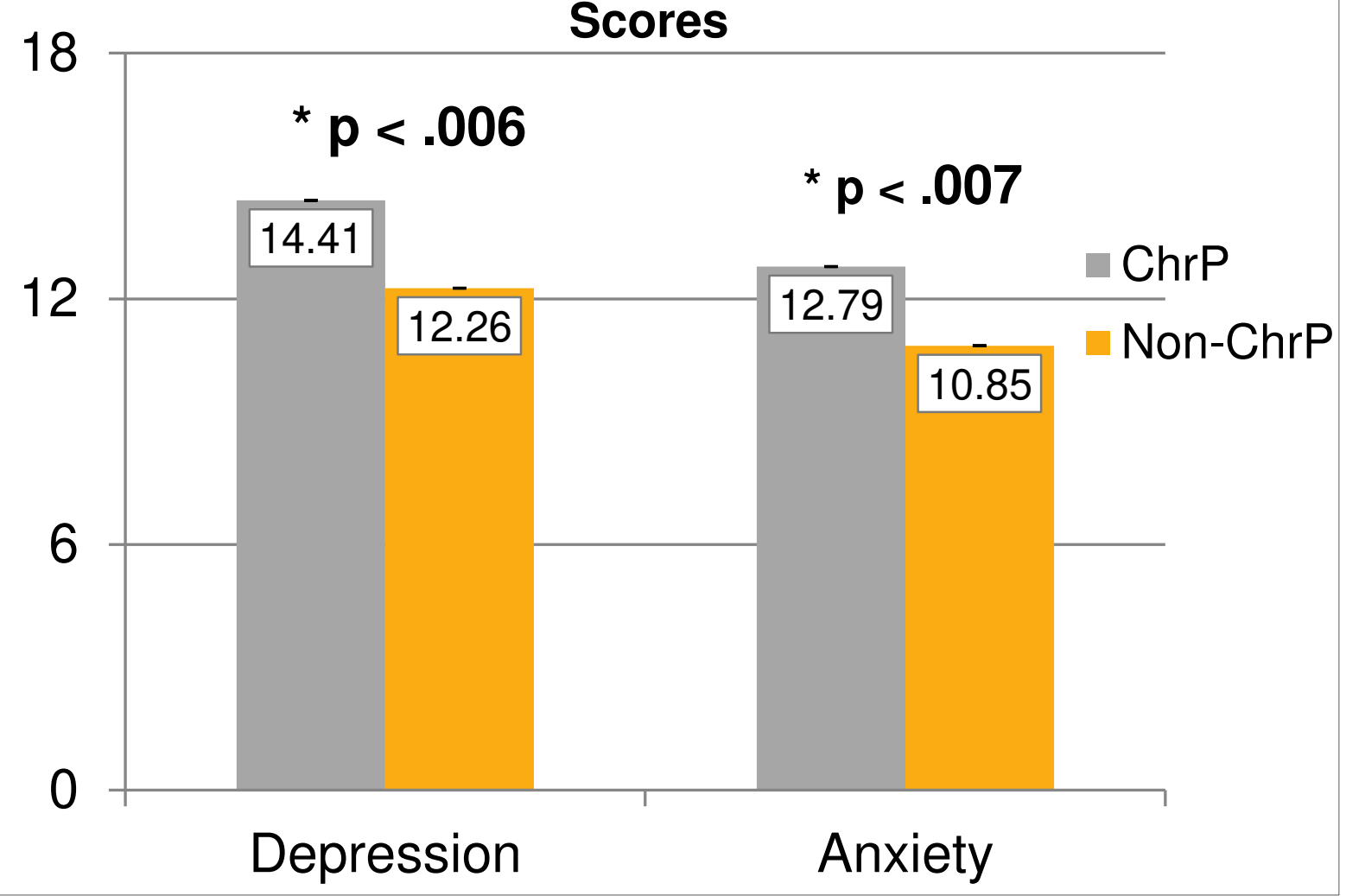

1 Chen \& Michalsen, 2017 BMJ State of the Art Reviews 1-19

2 Serdarevic et al., 2017 Current Opinion in Psychiatry 30, 238-46

3 Nahin, 2015 J Pain 16:769-80

4 Fishbein et al., 1986 Pain. 26(2); 181-97.
- Most prevalent CHPs included: massage therapy, chiropractic care, turmeric, ginger and acupuncture (see Table 1).

- $3.8 \%$ of ChrP women reported using cannabis for pain management

- ChrP women had higher mean PHQ depression scores than non-ChrP women (see Figure 1) and more of them scored in the moderately severe-to-severe range (PHQ score $\geq 15$ ) (see Figure 2).

- A similar pattern was seen for anxiety, with higher GAD means and more cases of severe anxiety (GAD-7 $\geq 15$ ) in ChrP group.

Summary:

- Chronic pain was prevalent and over half of ChrP women had been prescribed opioids.

- One third of ChrP women had used CHP, affirming the need for alternative pain management strategies

- High rates of recent clinical depression and anxiety in ChrP women warrant further study and affirm the need for screening and monitoring of such symptoms as part of ongoing medical care.

Table 1. CHP Used for Chronic Pain

\begin{tabular}{lll}
\hline & $\mathrm{N}$ & $\%$ \\
\hline Massage therapy & 21 & 10 \\
\hline Chiropractic & 14 & 6.7 \\
\hline Turmeric & 8 & 3.8 \\
\hline Acupuncture & 7 & 3.3 \\
\hline Ginger & 7 & 3.3 \\
\hline Tai Chi/Yoga & 6 & 2.9 \\
\hline Fish Oils/Omega & 4 & 1.9 \\
\hline Fatty Acids & &
\end{tabular}

Fig 2. Rates of Clinical Depression and Anxiety

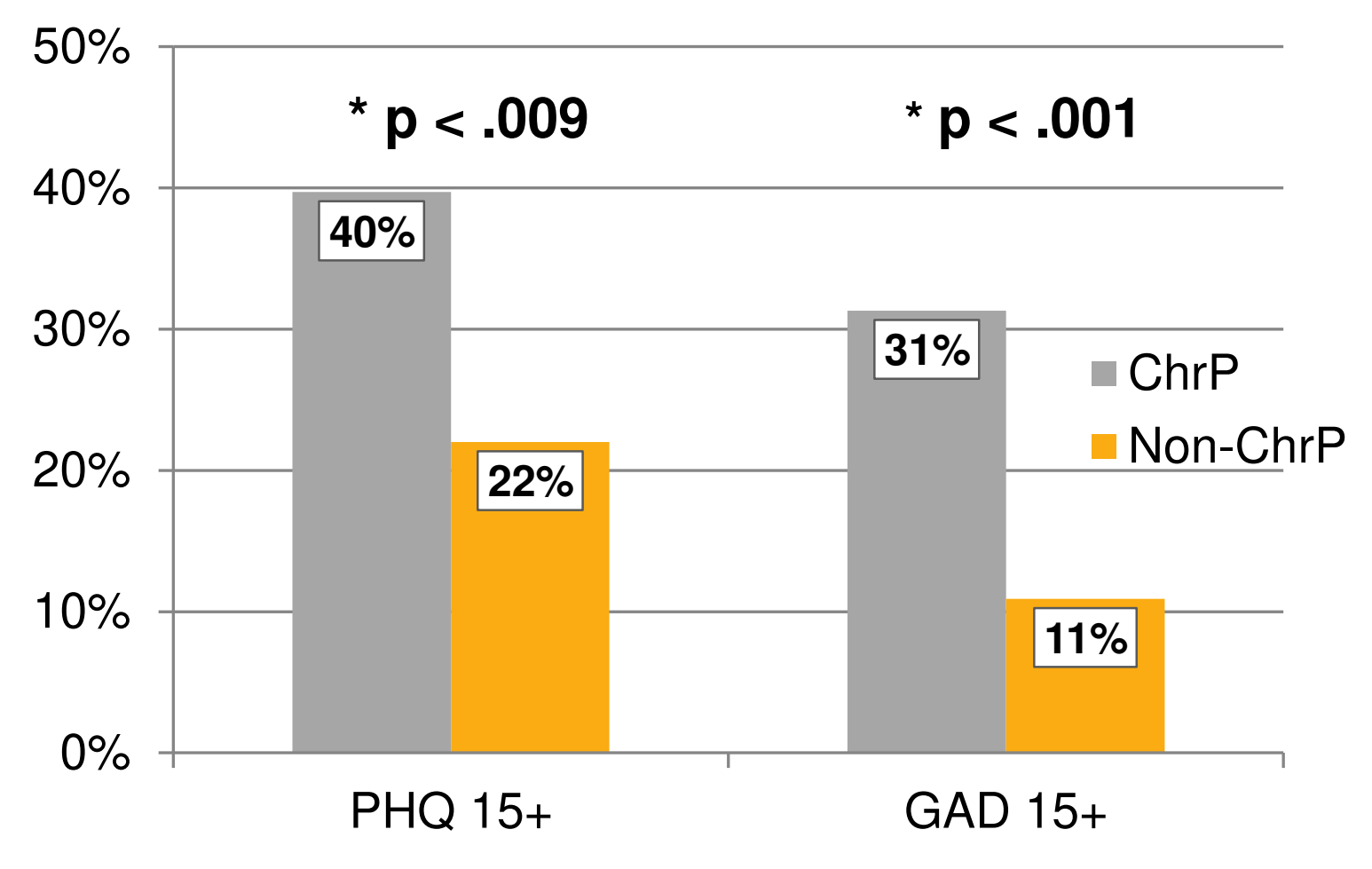

